Bamboo has been used by about 2.5 billion people, mostly for fibre, food and for handicrafts and building material, or for providing aesthetic and functional purposes to create good landscapes, especially in Asia, for thousands of years. Yet, its potential contribution to sustainable natural resource management has not been specially stressed. Bamboos are very important plants, both ecologically and economically. Bamboo forest is considered one of the best renewable resources on the planet, and is a sustainably-used resource once established. Its biological characteristics make it a perfect tool for solving many environmental problems, such as soil erosion control, water purification and conservation, carbon sequestration, recreation and so on.

Compared with other tall flowering plants, the pattern of bamboo growth may give an impression of high productivity, however, in fact, sometimes the "rapid growth" is a process of re-distribution of previously stored reserves. The entire bamboo sub-family have C3 photosynthesis lacking the $\mathrm{C} 4$ pathway and anatomy, thus the maximum possible productivity of bamboos is theoretically unlikely to be very high. Furthermore, the growth rate of bamboo is dependent on local habitat quality and climatic conditions.

Understanding the ecology of bamboo is unquestionably important, especially under the present global background of advocating low carbon economy and the potentially large ecological restoration works in many degraded tropical and subtropical areas, yet, so far, literature on the ecology of natural bamboo stands is meagre, and reports from plantation stands are rare. Bamboo has been neglected or ignored in the past by tropical foresters, who tend to concentrate on timber trees. Bamboo may have potential as a bioenergy or fiber crop, although some reports of its high productivity seem to be exaggerated. So far, literature on bamboo productivity is scarce, with most scattered reports coming from various parts of some Asian countries. Most bamboo currently has not been used scientifically and sustainably, since the rate of harvest from forest stands usually exceeds that of natural growth, and since the users usually do not have sufficient knowledge.

Bamboo resources in China are most abundant in the world. China is one of the earliest countries using the bamboo resources. It has long been the important forest resources in tropical and subtropical regions of China. According to the $6^{\text {th }}$ National Forest Resource Inventory of China, the present bamboo forest area in
China is about 500 million ha, accounting for $2.5 \%$ of forest area of China, and 39\% of the total area of bamboo of the world. There are 38 genera and 500 species of bamboo plant species in China, accounting for 36\% and $39 \%$ of the bamboo species of the world, respectively. Various aspects of bamboo have been studied in China relatively early and for the long-term, yet, these research results have not been summarized over time. The publication of the book Carbon Sequestration and Transformation in Bamboo Forest Ecosystem meets such a demand. The book is mainly based on the authors' research data in Zhejiang Province.

The book is divided into two parts. The first part reviews the main previous research results on carbon fixation and transformation in bamboo forest ecosystems. The second part is divided into 11 chapters. Chapter 1 summarizes the latest progresses in research on the carbon pool, carbon cycle, and carbon balance of forest ecosystems; Chapter 2 summarizes progress in research on the cultivation, management and biomass of bamboo; Chapter 3 and 4 deal with the spatial distribution of bamboo forests, and estimate the total biomass and carbon storage of bamboo in Zhejiang Province; Chapter 5 and 6 reveal the carbon accumulation and dynamics of bamboo forest with different management modes and different ages; Chapter 7 analyses the impacts of fertilization and floor cover on the evolution of soil organic carbon in bamboo forests; Chapter 8 compares soil active organic carbon pool of bamboo forests in subtropical area with those of other forest stands; Chapter 9 discusses and compares the abilities of soil carbon sequestration of bamboo, fir, and pine forests; Chapter 10 analyses the impacts of floor cover in winter on the rates of soil respiration in bamboo forests; Chapter 11 summarizes the main results of the present research on carbon fixation and transformation in bamboo forest ecosystems.

This book is suitable for scientists, teachers, students or other persons who are engaged in or interested in forest science, ecological science, soil science and relevant fields.

\section{DEZHI $^{1}$ AND QIN AILI ${ }^{2}$}

${ }^{1}$ Lab of Urbanization and Ecological Restoration of Shanghai; National Field Observation and Research Station in Tiantong Forest Ecosystem of Zhejiang; Department of Environmental Science, East China Normal University, 3663, Zhongshan Rd (N). Shanghai, China. 200062

${ }^{2}$ Shanghai Vocational and Technical College of Agriculture and Forestry, 658 Zhongshan 2 Road, Songjiang, Shanghai, China. 201600

\title{
Pitcher Plants of the Old World
}

By Stewart McPherson, 2009. Redfern Natural History Productions, 61 Lake Drive, Hamworthy, Poole, Dorset BH15 4LR, England, UK. xiii + 631 pages. (Volume 1); 768 pages (Volume 2). $£ 34.99$ (\$79.99) per volume plus shipping. Cloth.

Pitcher plants are intriguing in term of not only unusual forms, but also unique ways of nutrient acquisition. Pitcher plants include the largest and most spectacular of all carnivorous plants. They produce highly specialized foliage that takes the form of hollow, liquid-filled "pitchers", forming the extraordinary preytrapping mechanism to lure and prey upon insects, arthropods and other small animals. Contrast to most 
plants absorbing nutrients from the soil through their roots, carnivorous plants absorb nutrients from their animal prey through their leaves specially modified as traps. By trapping and digesting various invertebrates, and occasionally even small frogs and mammals, these plants whose most common habitat is in bogs and fens, where nutrient concentrations are low but water and sunshine seasonally abundant, can obtain some nutrients, thus they have the most bizarre adaptation strategies to unfavourable conditions, mostly low nutrient availability in wet, acid soils.

The carnivorous habit evolved independently in many plant lineages. In the Old World, there are two genera (families) of pitcher plants, i.e. Nepenthes and Cephalotus. Nepenthes occur across tropical areas of the western hemisphere, mainly in Southeast Asia, and consists of 120 species, including the largest of all carnivorous plant species which produce giant "pitcher" traps. Species of Nepenthes differs in the shape, size and colouration of its pitchers. Cephalotus grows only in Southeast Australia and produces small, purple pitchers the size of a thimble which are specialised towards the trapping of small prey.

Pitcher plants usually grow in harsh habitats which are usually inaccessible to humans. Thus, so far the knowledge about pitcher plants is not adequate. The newly published book Pitcher Plants of the Old World is up to date the most comprehensive overview and dependable and important reference book on pitcher plants of the Old World, and also the only publication dealing with the genus Nepenthes throughout its geographical range. The two volumes summarise the author's three-year research in the field. This 1399 page work contains 751 spectacular images. 120 species of Nepenthes, plus 5 incompletely diagnosed taxa are recognised, along with Cephalotus follicularis. Several species documented in this work have been discovered only very recently and, over 30 species have their photos published for the first time. All species in both genera of Nepenthes and Cephalotus of Old World are introduced in terms of the biology, ecology, diversity, distribution and conservation status and the various threats and practical means of conservation of every known species for the first time in detail, and also importantly high quality photographs illustrating the habitat, habit and pitchers (including forms on different parts of the plant where appropriate) are provided, These were taken by the author during 18 months of intensive and challenging fieldwork over a three-year period. The author also pays plenty of attention to the trapping mechanism and mutualistic fauna which inhabit trapping pitchers. Colour variants of the pitchers of many species are also illustrated. Cephalotus is treated in similar depth and, like Nepenthes, is illustrated with approximately 30 photographs, line drawings etc. Currently, many pitcher plant species are not in cultivation, and also there is often confusion among species, the author studied in detail, and photographed each species of Nepenthes and Cephalotus in the wild. This documentation has filled the lack of available information on these species, and is therefore of great scientific values.

At least some species of pitcher plants are on the verge of extinction in the wild, and some of them may have completely disappeared. The distribution of dozens of pitcher plant species usually are very limited, and sometimes the wild population may consist of just a few hundred plants. Furthermore they are faced with the serious risks of being poached, over-collected or having their habitat destroyed. The extraordinary value of these plants or the high horticultural interest made these risks even greater. It is highly likely that several species may become extinct over the next few years in the wild, if any ex situ or in situ conservation measures are not taken. The author's enthusiasm for the conservation and his commitment to habitat and ex situ conservation are shown throughout the two volumes, and some of the advice given. He also provides coverage of issues which seriously threaten the survival of many Nepenthes species, as well as information on cultivation of various species (highland Nepenthes are notoriously tricky to keep alive, even among those for whom carnivorous plants are more an obsession than a hobby).

Pitcher Plants of the Old World (Volume One) consists of the following chapters; Introduction, Carnivorous Plants of the World, The Pitcher Plants of the Old World, The Evolution of the Pitcher Plants of the Old World, Trapping Processes, Infauna, Nepenthes of Borneo and Nepenthes of Peninsular Malaysia and Indochina. Pitcher Plants of the Old World (Volume Two) comprises Nepenthes of the Philippines, Nepenthes of Sumatra and Java, Nepenthes of Sulawesi, Nepenthes of New Guinea and the Maluku Islands, Nepenthes of the Outlying Areas, Nepenthes Hybrids, Cephalotus follicularis, Habitat Loss and the Threat of Extinction and Cultivation and Horticulture, Appendix, Glossary, Bibliography, Index. The chapter on cultivation and horticulture provides historical information and up to date details on the conditions required to grow Nepenthes and Cephalotus successfully.

It is an excellent work to be enjoyed by plant enthusiasts and professional botanists in general, and is an essential book for carnivorous plant enthusiasts in particular.

\section{Li DeZHI ${ }^{1}$ AND QIN AILI ${ }^{2}$}

${ }^{1}$ Lab of Urbanization and Ecological Restoration of Shanghai; National Field Observation and Research Station in Tiantong Forest Ecosystem of Zhejiang; Department of Environmental Science, East China Normal University, 3663, Zhongshan Road (N), Shanghai, China. 200062

${ }^{2}$ Shanghai Vocational and Technical College of Agriculture and Forestry, 658 Zhongshan 2 Road, Songjiang, Shanghai, China. 201600 


\section{Erratum The Canadian Field-Naturalist 126(4)}

In response to the review of Contributions to the History of Herpetology. CFN 126(3): 344-345, the book's editor Kraig Adler pointed out (personal communication to FRC 12 May 2013): "Only one small correction. Mrs. Martof used a kitchen knife, not a gun. She told the police she slipped while cutting some pizza. But Bernie was stabbed up under his rib cage several times!"

\section{Erratum The Canadian Field-Naturalist}

It has come to our attention that sections of many of the book reviews by Li Dezhi and Qin Aili were copied from sources without attribution. The journal and the authors apologize for this oversight. 